\title{
The metastability of the mid-latitude Southern Hemisphere circulation
}

\author{
Terence J. O'Kane ${ }^{1} \quad$ James S. Risbey ${ }^{2}$ \\ Christian Franzke ${ }^{3} \quad$ Illia Horenko ${ }^{4}$ \\ Didier P. Monselesan ${ }^{5}$
}

(Received 9 October 2012; revised 17 April 2013)

\begin{abstract}
Observed changes in the metastability of the Southern Hemisphere $500 \mathrm{hPa}$ circulation are examined using non-stationary cluster analysis techniques. The cluster methodology is a purely data-driven approach for parametrisation whereby a multi-scale approximation to non-stationary dynamical processes is achieved through optimal sequences of locally stationary fast vector auto-regressive factor processes and some slow (or persistent) hidden process switching between them. Comparison is made with blocking indices commonly used in weather forecasting and climate analysis to identify dynamically relevant metastable regimes in the reanalysed $500 \mathrm{hPa}$ circulation. Our
\end{abstract}

http://journal.austms.org.au/ojs/index.php/ANZIAMJ/article/view/6177 gives this article, (C) Austral. Mathematical Soc. 2013. Published May 30, 2013, as part of the Proceedings of the 16th Biennial Computational Techniques and Applications Conference. ISSN 1446-8735. (Print two pages per sheet of paper.) Copies of this article must not be made otherwise available on the internet; instead link directly to this URL for this article. 
analysis characterises the metastable regime in reanalysed observational data sets prior to 1978 as positive and negative phases of a hemispheric mid-latitude blocking state with the Southern Annular Mode (SAM) associated with a transition state. Post 1978, SAM emerges as a true metastable state replacing the negative phase of the hemispheric blocking pattern. Trends in the hidden state frequency of occurrences correspond to declining blocking (coherent structures) and increasing strength of the SAM (zonal flow).

Subject class: 60

Keywords: cluster analysis, high dimensional data, climate

\section{Contents}

1 Introduction

C234

2 Methodology

C236

2.1 AIC: Akaike information criterion . . . . . . . . . . .

$\mathrm{C} 239$

2.2 Blocking indices . . . . . . . . . . . . . .

3 Results

C241

4 Conclusions

C243

References

C245

\section{Introduction}

The formation of quasi-stationary high pressure systems in the atmospheric mid-latitudes is often referred to as 'blocking'. The formation of a coherent blocking structure is necessarily associated with a reduction in the strength of the zonal circulation and a corresponding enhancement of the meridional motion. In the Southern Hemisphere ( $\mathrm{SH}$ ), mid-latitude blocks may persist 
on a time scale of the order of a week or longer. Charney and DeVore [3] first proposed that the observed multiple weather regimes are quasi-stationary or meta-stable states and that instability mechanisms are responsible for initiating transitions between said states. The physical mechanism which generates multiple equilibria is summarised as follows: strong zonal (eastward) jets in the mid-latitudes and the Coriolis effect create meridional temperature gradients, topography generates Rossby waves which in turn create drag on the flow, pushing it westward [3, 24]. Under suitable conditions, and for a particular value of the zonal wind, the waves might exhibit a resonant response causing the large scale flow to become locked near the resonant wind value. Thus, for particular values of zonal forcing, dissipation or topographic height the flow will settle into either a state with winds near the zonal forcing value or into one with winds near the resonant wind value, depending on the initial conditions. Assessing seven years of analyses and forecasts from the European Center for Medium Range Weather Forecasting operational archives, Tibaldi et al. [20] found only one preferred region for blocking in the $\mathrm{SH}$, around $180^{\circ}$ longitude, and that blocking in the SH was considerably more difficult to characterise than in the Northern Hemisphere (NH). Pook and Gibson [16] comprehensively reviewed the development of blocking indices specific to the SH, as used in operational weather prediction at the Australian Bureau of Meteorology. They noted that there are three well recognised SH blocking regions located to the east or southeast of the continents along latitude $45^{\circ} \mathrm{S}$ and that the Australian block (including the Tasman Sea and Southwest Pacific) is the most active. As blocking plays a critical role in determining precipitation in Southern Australia, correct identification of such events is of great importance [17].

Recently, Franzke et al. [7] applied a clustering method to systematically identify metastable atmospheric regimes in high dimensional datasets generated by a barotropic model and an atmospheric general circulation model. They employed a finite element clustering approach that decomposes the phase space into overlapping clusters while simultaneously estimating the most likely switching sequence among the clusters. The parameters of the 
clustering and switching were estimated by a finite element approach developed by Horenko [12]. The switching among the clusters is often described by a Markov transition matrix [7], while metastable regime behaviour can be assessed by inspecting the eigenspectrum of the associated transition probability matrix. Here we focus on the FEM-BV-VARX (finite element-bounded variation-vector auto-regressive factor) method $[10,11]$ in combination with the Akaike information criterion (AIC) to determine the optimal cluster state, as described in Section 2. O'Kane et al. [15] were the first to examine the relative merits of split-flow blocking indices and non-stationary cluster analysis for the SH.

The purpose of this article is to assess changes to the metastability of the $\mathrm{SH}$ atmospheric circulation based on transition sequences from the finite element clustering $[9,11]$ and in comparison to operational split-flow blocking indices [20, 23].

\section{Methodology}

In our approach we employ the FEM-BV-VARX method from Horenko [11]: a BV-regularized FEM-clustering based on the VARX-distance metric,

$$
\left\|x(t)-\mu(t)-\sum_{i=1}^{m} A_{i}(t)^{*} x(t-i \tau)-B(t) u_{t}\right\|,
$$

with the AIC (Section 7) to determine the optimal cluster state(s) K. We employ an empirical orthogonal function (EOF) decomposition prior to clustering to reduce the dimensionality, but do not formally include this decomposition as a part of the procedure. The FEM-BV-VARX algorithm does not simultaneously estimate a transition matrix for the cluster or meta-stable state evolution. Thus if one were to fit a Markov matrix, assuming stationarity, then the transition matrix and Markovianity must be computed and established a posteriori using a generator algorithm. In general, rather than calculate 
the transition matrix to determine the number of meta-stable states, we use the AIC and make no assumption of stationarity.

The FEM-BV-VARX [11] method simultaneously estimates the clusters (corresponding to regimes) and the most likely meta-stable state transitions between the clusters through the minimisation of an average clustering functional $L$ of a given time series $x_{t}$. This approach assumes that the dynamics of the observed variable of interest $x_{t}$ is influenced by the previous $m$ time-lagged values of the same variable (to describe the memory effects), some set of explicitly observed external factors $\mathfrak{u}_{\mathfrak{t}}$ and an unobservable (hidden) impact variable associated with regime transitions that strongly influences the observed variable.

Implicit in the FEM-BV-VARX approach is the assumption that the dynamics is approximated by a stochastic model of the general form

$$
x_{t}=\mu_{t}+A(t) \phi_{1}\left(x_{t-\tau}, \ldots, x_{t-m \tau}\right)+B(t) \phi_{2}\left(u_{t}\right)+C(t) \epsilon_{t} .
$$

Here, $\boldsymbol{\Theta}(\mathrm{t})=(\mu(t), A(t), B(t), C(t))$ is a vector of time dependent model parameters, $\epsilon_{\mathrm{t}}$ is a stochastic variable describing the noise, $\phi_{1}\left(x_{\mathrm{t}-\tau}, \ldots, x_{\mathrm{t}-\mathrm{m} \tau}\right)$ is some (in general) non-linear function which connects the earlier observations $x_{t-\tau}, \ldots, x_{t-m \tau}, \phi_{2}\left(u_{t}\right)$ is an external factor function, and $\mathrm{C}(t)$ couples the unobserved scales (modeled as a statistically independent and identically distributed noise process with zero expectation) to the analysed time series. As demonstrated by Horenko [9], time dependence of the model parameters $\boldsymbol{\Theta}$ is induced by the influence of the unresolved scales and leads to non-stationary regime transition behaviour in many realistic systems.

The FEM-BV-VARX method aims to identify the time dependent optimal parameters $\boldsymbol{\Theta}(t)$ as a convex linear combination of time independent parameters $\theta_{i}$ for $i=1 \ldots, K$ with time dependent linear combination coefficients $\gamma_{i}(t)$. In the context of the FEM-BV-VARX, functions $\gamma_{i}(t)$ are interpreted as the probabilities that the given observation $x_{t}$ at time $t$ is best explained by a VARX model with constant model parameters $\theta_{i}$. Thus, for an a priori given number of clusters $\mathrm{K}$, fixed given time series $\boldsymbol{x}_{\mathfrak{t}}$ and $\boldsymbol{u}_{\mathrm{t}}$, and fixed maximal time lag $\mathrm{m}$, the FEM-BV-VARX methodology minimises the distance of the 
actual trajectory (in an appropriate metric $\mathrm{g}\left(\mathrm{x}_{\mathrm{t}}, \mathfrak{u}_{\mathrm{t}}, \theta_{\mathrm{i}}\right)$ ) to one of the $\mathrm{K}$ model clusters at time $t$. This means that we are looking simultaneously for the cluster locations (defined by the cluster probabilities $\gamma_{i}(t), i=1, \ldots, K$ ) and the time evolution of the system in the space spanned by the model clusters defined by the parameters $\theta_{i}$, and where $\Gamma(t)=\gamma_{1}(t), \gamma_{2}(t), \ldots, \gamma_{k}(t)$ defines the model affiliation sequence or Viterbi path.

The method considers the clustering of possibly non-stationary multidimensional data $x_{t} \in \mathbb{R}^{\mathrm{d}}$ as a minimisation problem,

$$
\mathrm{L}(\boldsymbol{\Theta}, \Gamma)=\sum_{\mathrm{t}=0}^{\mathrm{T}} \sum_{i=1}^{\mathrm{K}} \gamma_{i}(\mathrm{t}) \mathrm{g}\left(\mathrm{x}_{\mathrm{t}}, \mathfrak{u}_{\mathrm{t}}, \theta_{\mathrm{i}}\right) \rightarrow \min [\Gamma(\mathrm{t}), \boldsymbol{\Theta}(\mathrm{t})],
$$

subject to constraints

$$
\sum_{i=1}^{K} \gamma_{i}(t)=1 \quad \text { for all } t \in[0, T]
$$

and

$$
\gamma_{i}(t) \geqslant 0 \quad \text { for all } t \in[0, T] \text { and } i=1, \ldots, K,
$$

where we want to minimise the object L. The corresponding cluster distance functional characterises how well a given observation $x_{t}$ at time $t$ is described by a given model $i$ with parameters $\theta_{i}$ [9]. One incorporates additional information into the optimisation, such as some persistency assumptions of functions in space $\Gamma($.$) , and then applies a finite Galerkin time discretisation$ of this infinite dimensional Hilbert space. For example, for a given observation time series one can impose the constraint of the limited (that is bounded) temporal variation of the underlying statistical parameters $\boldsymbol{\Theta}(\mathrm{t})[8,9]$.

The optimisation problem is now solved by a finite element approach $[9,10$, for algorithm details] using principal components of the EOFs, as described. The persistency constraint $C$ bounds the persistency of the function $\gamma_{i}$ via 
the norm

$$
\begin{aligned}
\left|\gamma_{i}\right|_{B V(0, T)} & =\sum_{t=0}^{T-1}\left|\gamma_{i}(t+1)-\gamma_{i}(t)\right|=\left\|D \gamma_{i}^{\dagger}\right\|_{1} \leqslant C, \\
D & =\left[\begin{array}{cccc}
-1 & 1 & \ldots & 0 \\
0 & -1 & \ldots & 0 \\
\vdots & \vdots & \vdots & \vdots \\
0 & \ldots & -1 & 1
\end{array}\right],
\end{aligned}
$$

where $i=1, \ldots, K, \gamma_{i}=\left[\gamma_{i}(1), \ldots, \gamma_{i}(T)\right] \in \mathbb{R}^{\top}, \dagger$ is the transposition operation and $\|\cdot\|_{1}$ is the 1 -norm. The scalar persistency parameter $\mathrm{C}$ measures the maximal number of transition between the local model $i$ and all other models in the time interval $(0, T)$.

\subsection{AIC: Akaike information criterion}

In the context of non-stationary inference, it is appropriate to use the AIC to determine the right order parameters of the VARX model, that is the memory depth $\mathrm{m}$, the number of clusters $\mathrm{K}$ and the optimal BV-persistency $\mathrm{C}[11,15]$. To select the proper order parameters (and the optimal functional for external factors $\phi_{2}\left(\mathfrak{u}_{\mathfrak{t}}\right)$ in (2)) for a given persistency parameter value [11] the AIC is defined as

$$
\mathrm{AIC}=-2 \log \mathrm{L}_{\max }+2 \mathrm{M},
$$

where $L_{\max }$ is the maximum likelihood achievable by the model and $M$ is the number of free parameters. The lowest AIC is preferred. The resulting optimal Viterbi path provides a natural method for generating the climatology of a particular cluster state sequence. Cluster states are constructed by first assigning a model affiliation to each data point in the time series of anomalies according to the Viterbi path sequence $\Gamma(t)$. Then all anomalies for each given cluster state assignation are averaged. The averaged state is the composite or cluster state. 


\subsection{Blocking indices}

We are interested in the utility of the FEM-BV-VARX with regard to its ability to identify systematic changes in the regime states of the climate system. The dynamics of the mid-latitude SH atmosphere are characterised by the presence of regimes represented by the SAM and high-low blocking dipoles. To identify said regime states the FEM-BV-VARX must be able to accurately identify SH blocking. Meteorologists typically employ split-flow blocking indices to identify mid-latitude blocking. Such indices are also used to assess climate general circulation model performances [19] and to develop blocking climatologies [16]. Pook and Gibson [16] discussed in some detail the respective definitions of SH blocking and the development of the modern blocking index used at the Australian Bureau of Meteorology (BoM), as developed by Wright [23]. This blocking index, which we refer to as the Bom index, is defined as

$$
0.5\left(\mathrm{U}_{25}+\mathrm{U}_{30}-\mathrm{U}_{40}-2 \mathrm{U}_{45}-\mathrm{U}_{50}+\mathrm{U}_{55}+\mathrm{U}_{60}\right)>0,
$$

where $\mathrm{U}_{\mathrm{y}}$ represents the zonal component of the mean $500 \mathrm{hPa}$ wind (five day averaged) at latitude $y$. The BoM index detects blocking whenever it is positive. Large values indicate strong high and low latitude westerly winds or that the mid-latitude westerly flow is weak. O'Kane et al. [15] considered a related measure of SH blocking, originally developed by Tibaldi et al. [20], which depends on mid- and high latitude geo-potential height gradients.

In this study we examine the National Center for Environmental Prediction (NCEP) and the National Center for Atmospheric Research (NCAR) reanalysis data set using $500 \mathrm{hPa}$ geopotential height fields in the $\mathrm{SH}$, covering the period January 1948 through December 2009. Due to the dependence of SH observations on satellite data, reanalysis is not considered very reliable before about 1979. However, we choose to include an analysis of the complete data set as we consider only large scale hemispheric features and consider reanalysis to be a reasonable best guess. The FEM-BV-VARX algorithm requires continuous data and so we use daily data over a 60 year period. This approach is different to almost all other clustering studies which focus on the NH winter season. 
01 annual ncep

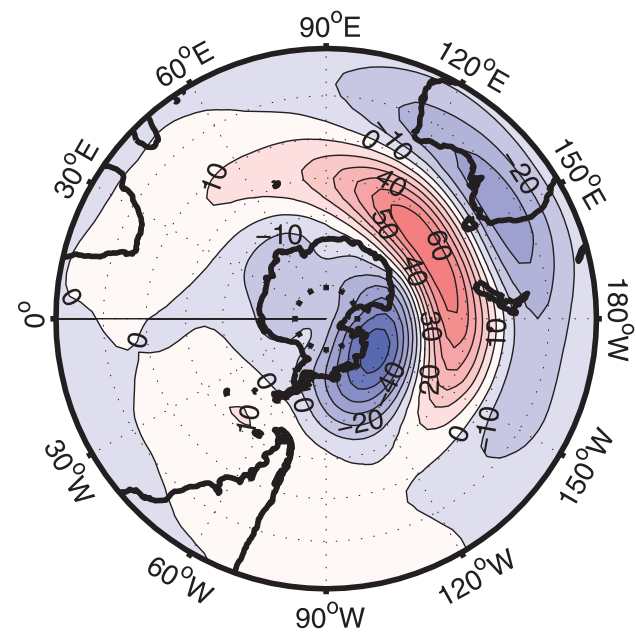

02 annual ncep

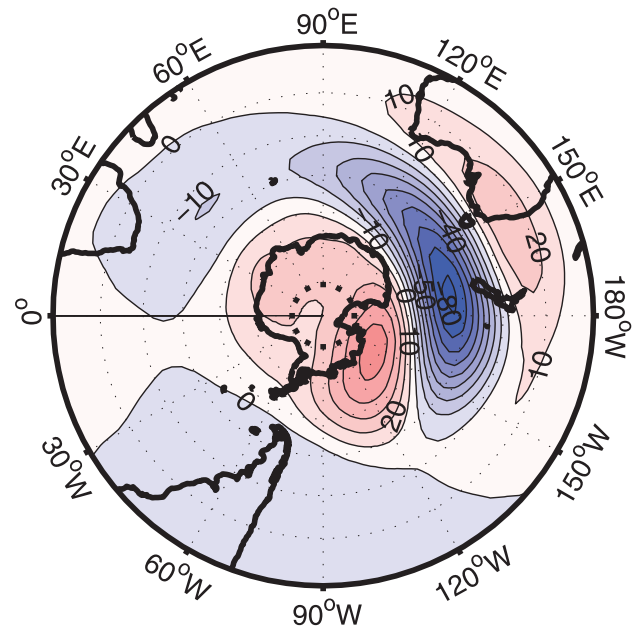

Figure 1: NCEP 500 hPa 1948-2010: composite states using the BoM blocking index. Australian region (longitude sector $110^{\circ} \mathrm{E}-210^{\circ} \mathrm{E}$ ) averaged over all seasons.

We first subtract a smooth annual cycle from the data by computing the mean annual cycle by taking the calendar average for each day. We also find that smoothing the noisy annual cycle via a running mean has little effect on the results.

\section{Results}

We calculate composites from the Bom blocking index by considering all instances where the BoM index is greater than one standard deviation above the mean to be in the $(+1)$ state and all instances where the Bom index is less than one standard deviation below the mean to be in the $(-1)$ state. This is carried out for both the SH and the Australian blocking region, defined here as the longitude sector $110^{\circ} \mathrm{E}-210^{\circ} \mathrm{E}$ (Figure 1). 
01 annual ncep

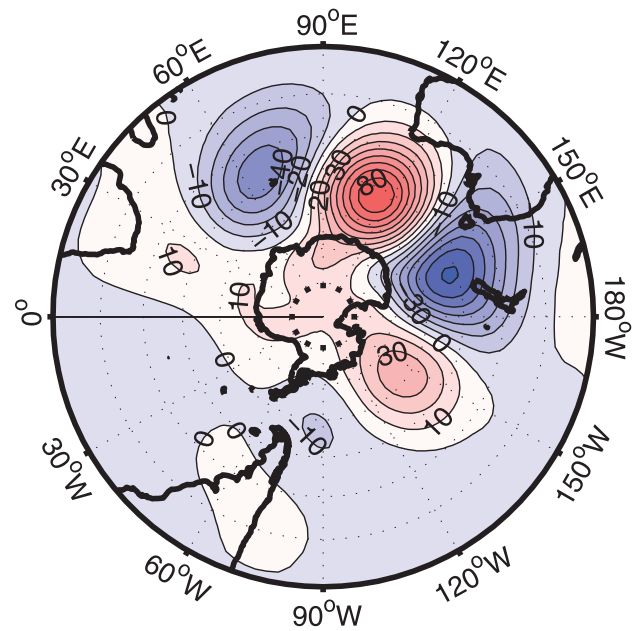

02 annual ncep

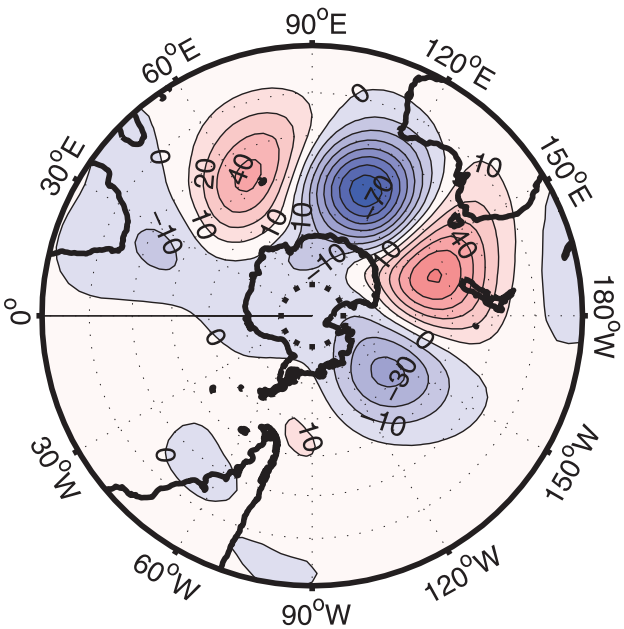

Figure 2: NCEP 500 hPa 1948-2010: composite states using the FEM-BV-VARX Viterbi paths. Australian region (longitude sector $110^{\circ} \mathrm{E}-210^{\circ} \mathrm{E}$ ) averaged over all seasons.

Although the BoM index and FEM-BV-VARX Viterbi paths (not shown) are similar, there are manifest differences between the BoM and FEM-BV-VARX composite states. Ideally, blocking composites from any method should resemble the 'canonical' block. For the Australian region this typically consists of anticyclones in the latitude band $35^{\circ} \mathrm{S}-60^{\circ} \mathrm{S}$, most commonly in the Tasman sea but also occurring in the Western Pacific, Great Australian Bight and eastern Indian Ocean [1, 21]. While splitting of the basic westerly flow implies a high-low dipole, blocking in the Australian region is typified by a larger and more conspicuous high [22] or sequence of highs [1]. As the FEM-BV-VARX method is not descriptive and makes no assumptions about the structure of blocks there is no a priori guarantee that the cluster composites will resemble structures that look like blocks. However, for the Australian region the FEMBV-VARX produces composites with coherent structures that are confined to the mid-latitude jet with maxima in the locality of the Tasman sea (Figure 2). More generally, the global FEM-BV-VARX composites [15, Figures 2 and 3] 
show structures immediately recognisable as coherent blocked states and do match the features of blocking in the SH; that is a three wave blocking structure in the SH with nodes in the dominant blocking regions [21].

In contrast, for the Australian region the BoM split flow index generates blocking composite states with anomalies across a large latitudinal band that straddles both the subtropical and polar jets, consistent with a splitting of the flow (Figure 1). For the global Bom index composites [15, Figure 9], the three wave structure is weak and the nodes are displaced from the regions usually associated with persistent height anomalies in the literature [13, 18] and observed in individual case studies [14, 21]. Figure 3 shows the pivot point in residence length for the NCEP atmospheric reanalysis data at 500 geopotential height, marking the transition from a SH weather regime dominated by strong mid-latitude blocking (three wave hemispheric patterns) to one in which the SAM (zonal hemispheric flow) is increasingly dominant.

\section{Conclusions}

The meta-stable states of the SH over the NCEP reanalysis period show that post 1978 there has been a significant decline in blocking over the summer months and during spring. However, post 2000 there is little evidence of trends in autumn and winter. Similar trends, consistent with the satellite period, are also observed over the full reanalysis period, and while most significant in summer and spring, they are also observable in winter and autumn. For the satellite period, the FEM-BV-VARX metastable states are clearly SAM and a hemispheric blocking state with positive anomalies in the three $\mathrm{SH}$ blocking regions. Taken over the entire reanalysis period, the FEM-BV-VARX cluster states are positive and negative blocking phases. Summer averaged transition states for both periods resemble SAM. Taken as a whole, these results indicate that there has been a fundamental change in the regime states of the SH atmospheric circulation whereby the negative blocking pattern has been progressively replaced over time by SAM as a metastable state and that 


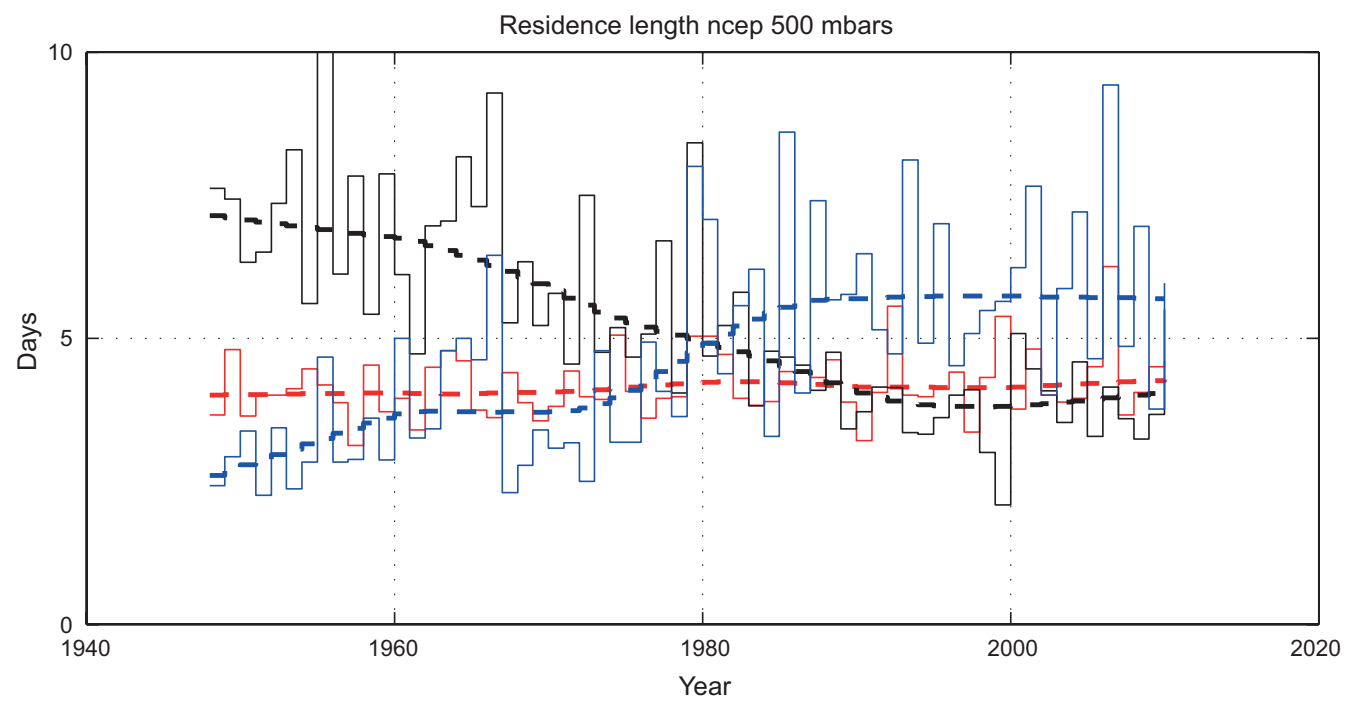

Figure 3: Residence length in positive (black dashed), negative (blue dashed) and transition (red dashed) phases of a hemispheric mid-latitude blocking state (three wave hemispheric pattern), obtained from a cluster analysis of SH 500 geopotential height.

SAM is intensifying, while blocking is in decline both in terms of occurrence and persistency.

We showed qualitative agreement between cluster affiliation sequences (Viterbi paths) and split-flow blocking indices commonly used in operational meteorology and to construct blocking climatologies. Closer inspection revealed significant differences between the BoM index and FEM-BV-VARX composite states, both hemispheric and for the Australian region. The BoM index composite states were found to be representative of split flow while the FEMBV-VARX composites capture localised coherent anomalies associated with blocking. For the Australian region, the Tibaldi index and criteria states closely match those using the Bom index with positive anomalies to the southeast of New Zealand and at the higher latitudes. FEM-BV-VARX composites 
were found to have positive anomalies in the mid-latitudes between Australia and New Zealand in regions where blocks are, on average, most typically found.

In contrast to standard clustering methods (for example, approaches related to K-means [4], fuzzy C-Means [2], Gaussian mixture models [5] or hidden Markov models [6]), the FEM-BV-framework does not rely on (strong) a priori probabilistic assumptions about the data. Additionally, FEM-BV is a more well posed numerical method than the standard clustering approaches since the BV constraint on the switching process limits the number of possible solutions that are attained as it is more robust with respect to the possibility of being trapped in the local optimum of the respective cluster quality functional. Moreover, deployment of the adaptive finite element methods (standard approach for a numerical solution of partial differential equations) in the FEM-BV context enables adaptive and parallelised clustering of the very large amounts of data common in practical applications. This method was successfully applied to identify atmospheric blocking events in the Northern [9] and Southern [15] Hemispheres from the slow temporal changes of the statistical model parameters describing the dynamical interactions of geopotential pressure values with some global atmospheric factors (for example $\mathrm{CO}_{2}$ concentration, solar activity).

\section{References}

[1] P. G. Baines. A survey of blocking mechanisms, with application to the Australian region. Aust. Met. Mag., 31:27-36, 1983. http://www.bom.gov.au/amoj/docs/1983/baines.pdf C242

[2] J. C. Bezdek. Pattern recognition with fuzzy objective function algorithms. Plenum Press, New York, ISBN 0-306-40671-3, 1981. C245 
[3] J. G. Charney and J. G. DeVore. Multiple flow equilibria in the atmosphere and blocking. J. Atmos. Sci., 36:1205-1216, 1979. doi:10.1175/1520-0469(1979)036<1205:MFEITA > 2.0.CO;2 C235

[4] A. Dawson, T. N. Palmer, and S. Corti. Simulating regime structures in weather and climate prediction models. Geophys. Res. Lett., 39:L21805, 2012. doi:10.1029/2012GL053284 C245

[5] A. P. Dempster, N. M. Laird, and D. B. Rubin. Maximum likelihood from incomplete data via the em algorithm. Journal of the Royal Statistical Society Series B, 39:1-38, 1977. http://www. jstor.org/stable/2984875 C245

[6] C. Franzke, D. T. Crommelin, A. Fischer, and A. J. Majda. A hidden markov model perspective on regimes and metastability in atmospheric flows. J. Climate, 21:1740-1757, 2008. doi:10.1175/2007JCLI1751.1 C245

[7] C. Franzke, I. Horenko, A. J. Majda, and R. Klein. Systematic metastable atmospheric regime identification in an agcm. J. Atmos. Sci, 66:1997-2012, 2009. doi:10.1175/2009JAS2939.1 C235, C236

[8] I. Horenko. On robust estimation of low-frequency variability trends in discrete markovian sequences of atmospheric circulation patterns. J. of Atmos. Sci., 66(11):2059-2072, 2009. doi:10.1175/2008JAS2959.1 C238

[9] I. Horenko. Finite element approach to clustering of multidimensional time series. SIAM J. Sci. Comput., 32:62-83, 2010. doi:10.1137/080715962 C236, C237, C238, C245

[10] I. Horenko. On clustering of non-stationary meteorological time series. Dynamics of Atmospheres and Oceans, 49:164-187, 2010. doi:10.1016/j.dynatmoce.2009.04.003 C236, C238

[11] I. Horenko. On the identification of nonstationary factor models and their application to atmospheric data sets. J. Atmos. Sci., 67:1559-1574, 2010. doi:10.1175/2010JAS3271.1 C236, C237, C239 
[12] I. Horenko. Nonstationarity in multifactor models of discrete jump processes, memory and application to cloud modeling. J. Atmos. Sci., 68(7):1493-1506, 2011. doi:10.1175/2011JAS3692.1 C236

[13] D. A. Jones and I. Simmonds. A climatology of Southern Hemisphere anticyclones. Climate Dynamics, 10:333-348, 1994. doi:10.1007/BF00228031 C243

[14] P. F. Noar. Numerical modelling of blocking, with reference to June 1982. Aust. Met. Mag., 31:37-49, 1983.

http://www. bom.gov.au/amoj/docs/1983/noar.pdf C243

[15] T. J. O'Kane, J. S. Risbey, C. Franzke, I. Horenko, and D. Monselesan. Changes in the metastability of the mid-latitude southern hemisphere circulation and the utility of non-stationary cluster analysis and split flow blocking indices as diagnostic tools. J. Atmos. Sci., 70:824-842, 2013. doi:10.1175/JAS-D-12-028.1 C236, C239, C240, C242, C243, C245

[16] M. Pook and T. Gibson. Atmospheric blocking and storm tracks surfing SOP-1 of the FROST Project. Aust. Met. Mag., pages 51-60, 1999. http://www.bom.gov.au/amoj/docs/1999/pook.pdf C235, C240

[17] J. S. Risbey, M. J. Pook, P. C. McIntosh, M. C. Wheeler, and H. H. Hendon. On the remote drivers of rainfall variability in Australia. Mon. Wea. Rev., 137(10):3233-3253, 2009. doi:10.1175/2009MWR2861.1 C235

[18] M. R. Sinclair. A climatology of anticyclones and blocking in the Southern Hemisphere. Mon. Wea. Rev., 124:245-263, 1996. doi:10.1175/1520-0493(1996)124<0245:ACOAAB > 2.0.CO;2 C243

[19] S. Tibaldi. Low-frequency variability and blocking as diagnostic tools for global climate models. Proc. NATO Advanced Research Workshop on Prediction of Interannual Climate Variations, NATO-ASI:173-182, 1993. C240

[20] S. Tibaldi, E. Tosi, A. Navarra, and L. Pedulli. Northern and southern hemisphere seasonal variability of blocking frequency and predictability. 
Mon. Wea. Rev., 122:1971-2003, 1994.

doi:10.1175/1520-0493(1994)122<1971:NASHSV>2.0.CO;2 C235, C236, $\mathrm{C} 240$

[21] K. E. Trenberth and K. C. Mo. Blocking in the Southern Hemisphere. Mon. Wea. Rev., 113:3-21, 1985. doi:10.1175/1520-0493(1985)113<0003:BITSH > 2.0.CO;2 C242, C243

[22] A. D. F. Wright. Blocking action in the Australian region. Bur. Met., Australia, Tech. Report 10:27pp, 1974. C242

[23] W. J. Wright. Seasonal climate summary southern hemisphere (autumn 1993): a second mature ENSO phase. Aust. Met. Mag., 43:205-221, 1994. http://www.bom.gov.au/amoj/docs/1994/wright.pdf C236, $\mathrm{C} 240$

[24] M. Zidikheri, J. S. Frederiksen, and T. J. O'Kane. Multiple equilibria and midlatitude atmospheric blocking: a re-examination. World Scientific Lecture Notes in Complex Systems Vol. 6, Frontiers in turbulence and coherent structures, pages 59-86, 2007. C235

\section{Author addresses}

1. Terence J. O'Kane, CSIRO Marine and Atmospheric Research, Hobart, Tasmania 7001, Australia.

mailto:terence.okane@csiro.au

2. James S. Risbey, CSIRO Marine and Atmospheric Research, Hobart, Tasmania 7001, Australia.

3. Christian Franzke, British Antarctic Survey, Cambridge, United Kingdom.

4. Illia Horenko, Universita della Svizzera Italiana, Lugano, Switzerland. 
5. Didier P. Monselesan, CSIRO Marine and Atmospheric Research, Hobart, Tasmania 7001, Australia. 